\title{
Aspectos Determinantes del Éxito Académico de Estudiantes Universitarios
}

\author{
Karla Soria-Barreto y Sergio Zúñiga-Jara \\ Universidad Católica del Norte - Coquimbo, Escuela de Ciencias Empresariales. Larrondo 1281, Coquimbo- \\ Chile (e-mail: ksoria@ucn.cl; sz@ucn.cl)
}

Recibido May. 14, 2014; Aceptado Jul. 23, 2014; Versión final recibida Ago. 13, 2014

\begin{abstract}
Resumen
En este trabajo se analizan algunos aspectos determinantes del éxito académico de estudiantes universitarios. Se considera como medida del éxito alcanzado que el estudiante logre ser titulado y egresar, mientras que se considera fracaso a las causales de eliminación, abandono de estudios o traslado. Para esto se elaboró una base de datos de 564 estudiantes universitarios, con 13 variables explicativas clasificadas en tres categorías: características personales, educación media, resultados de la prueba de aptitud académica. Como resultado de aplicar dos modelos logit, las principales variables que resultaron determinantes estadísticamente en el éxito de los estudiantes fueron, en primer lugar las notas de enseñanza media, en segundo lugar el puntaje obtenido en la Prueba de Aptitud Académica de Matemáticas y finalmente el número de años de desfase entre el año de egreso de la enseñanza media y el año de ingreso a la universidad.
\end{abstract}

Palabras clave: éxito académico, rendimiento académico, desempeño estudiantil, predicción del rendimiento

\section{Determining Aspects of Academic Success of University Students}

\begin{abstract}
In this study some determining aspects of the academic success of university students are analyzed. In this study the measure of success is the graduation of a student while student's removal, transfer or dropout from the university is considered failure. To compare students' success/failure rates, a database of 564 university students was used. The database included 13 explanatory variables classified into three categories: student's personal characteristics, high school grades, and university admission test scores. Results using logit models, show that the main variables predicting academic success are: first, high school grades; second, scores obtained by the student on the math portion of the university admission test; and finally, the gap in terms of the number of years between the year of graduation from high school and the year of university enrollment.
\end{abstract}

Keywords: success performance, academic performance, student performance, performance prediction 


\section{INTRODUCCION}

Uno de los desafíos más relevantes que enfrenta la investigación en docencia universitaria es explicar por qué algunos estudiantes universitarios tienen muy buen desempeño, mientras que otros no logran terminar sus estudios. La deserción de los estudiantes universitarios es una problemática que ha sido abordada ya desde la década de los setenta, en un comienzo por estudiosos de las mismas universidades que veían como cada vez más estudiantes universitarios no terminaban su proceso educacional. Además, la deserción universitaria y el retraso en los estudios son dos problemas de alcance internacional como lo mencionan Huesca y Castaño (2007) para Europa, donde el crecimiento de la deserción se ha acrecentado últimamente. En periodos recientes han emergido un gran número de estudios intentando explicar las causas del éxito-fracaso universitario. Romo y Fresán (2001) identifican como elementos explicativos la falta de personalidad y madurez intelectual de los estudiantes, así como la falta de conocimientos y habilidades previas necesarias para realizar estudios superiores. La intención y el compromiso son para Tinto (1993) los dos atributos que destacan como causas primarias de la deserción, y que cuánto más integradoras son esas experiencias, más probabilidades tiene el alumno de permanecer en la institución.

Ishitani y Snider (2006) analizan una muestra de más de 4.000 estudiantes norteamericanos participantes de programas de preparación para la universidad para determinar la probabilidad de deserción al ingresar a la universidad. Dentro de los resultados encontrados está que, asistir a estos programas de preparación, disminuye la probabilidad de salida de los estudiantes, más aún si los padres están comprometidos con los estudios de sus hijos. Asimismo, el ingreso del hogar arrojó un efecto significativo y directo en la deserción, es decir, los estudiantes provenientes de hogares con mayores ingresos tenían mayor probabilidad de completar sus estudios. Por otro lado, aquellos estudiantes que reciben apoyo financiero aumentan su probabilidad de deserción en un $89 \%$ para el segundo año de estudios. Los alumnos que son primera generación en asistir a la universidad tienen un $82 \%$ mayor de probabilidad de desertar que aquellos estudiantes cuyos padres han pasado por estudios universitarios. Finalmente, también concluyeron que durante el primer año de universidad, su historial académico es un factor determinante para abandonar la carrera, aquellos estudiantes con bajo promedio académico, tendrán mayor probabilidad de abandonar sus estudios. Duarte y Galaz (2006) identificaron variables predictivas del desempeño académico analizando antecedentes escolares, socio demográficos, de auto percepción, habilidades académicas, redes familiares y sociales, salud y elección de carrera. Se observó que el rendimiento académico durante el bachillerato fue el indicador que mejor predijo el desempeño académico, de modo que el promedio de calificaciones del bachillerato es un predictor considerablemente más fuerte que los exámenes de admisión.

En la misma línea anterior, Chain et al. (2003) concluyeron que la trayectoria universitaria se asociaba de mejor manera a la trayectoria del bachillerato, incluido el nivel de calificaciones. Vásquez y Cabrera (2004) encontraron que manteniendo constantes las variables demográficas, académicas preuniversitarias y socioeconómicas, la decisión de persistencia/deserción es el resultado de cuan adecuada o inadecuada fue la integración académica o social. También, que a mayor índice académico (desempeño durante el primer año de universidad), menor es el riesgo de abandonar los estudios universitarios. Herrera et al. (1999) encuentran que las variables que contribuyen, en mayor medida, a explicar el rendimiento son, en orden de importancia, el rendimiento anterior al ingreso en la universidad, los hábitos de estudio, la asistencia a clase, la satisfacción por la carrera elegida, la edad en sentido negativo, los estudios de la madre, la actitud hacia la universidad-profesores-compañeros, la auto eficacia y estudios del padre. Vásquez y Cabrera (2004) encuentran que del grupo de variables académicas preuniversitarias, el Índice Académico de Escuela Superior (IES) fue la única que arrojó un efecto significativo sobre la probabilidad de éxito en completar el bachillerato, y que de las variables relacionadas con el capital social y cultural, sólo la educación formal del padre es significativa en la probabilidad de éxito. Ibarra y Michalus (2010) encontraron que las variables significativas del rendimiento académico son el promedio de calificaciones del nivel medio, el tipo de Institución donde se cursó estos estudios y el número de asignaturas aprobadas en el primer año de carrera, siendo este último factor el más relevante. Albalate et al. (2011) constatan que el número de horas de estudio y la asistencia en clase influyen en los resultados obtenidos por los estudiantes, y que la variable que resulta claramente significativa hace referencia a los conocimientos previos de los alumnos.

Martínez-Padilla y Pérez-González (2008) determinaron tres variables relevantes para predecir el grado de éxito o fracaso de estudiantes mexicanos para el examen nacional de egreso de la licenciatura en ingeniería. Encontraron que las variables relacionadas con la trayectoria académica que mayor efecto tiene en la estimación de dicho desempeño corresponden al promedio general alcanzado en la enseñanza media, el rendimiento académico, y la cantidad de materias que fueron reprobadas durante su permanencia en la universidad.

En el caso de Chile, el proceso evolutivo de la educación durante las últimas décadas se caracteriza por el aumento en el número de matrículas, especialmente de los quintiles de menores ingresos. Bastías et al. 
(2000) encuentran que la variable más importante para explicar la trayectoria óptima es la nota de enseñanza media, seguida por el puntaje de la prueba específica de biología, estudios secundarios en la Región Metropolitana y los estudios universitarios previos, en ese orden. En varios estudios se ha analizado la validez predictiva de la Prueba de Selección Universitaria (PSU) por el Comité Técnico Asesor, H. Consejo de Rectores de las Universidades Chilenas (2006 y 2010), encontrando que las notas de enseñanza media son el factor más eficaz para predecir el rendimiento de los alumnos que ingresan a la universidad. Contreras et al. (2009) concluyen que en Chile el haber estado entre los mejores estudiantes de la escuela de egreso (ranking) implica un mejor desempeño universitario, superando a las pruebas de selección (PSU) e incluso que las notas de enseñanza media. Reyes y Torres (2009) concluyen que las notas de enseñanza media (NEM) y las Pruebas de Selección Universitaria (PSU), tiene el mayor peso relativo dentro en todas las especificaciones de regresión que ellos analizaron, y que las variables del entorno siguen en importancia.

Perez et al. (2011) también reportan que las NEM son un mejor predictor de características cognitivas y actitudinales de los estudiantes, siendo importantes para su éxito en la universidad, y cuestionando la alta ponderación que los procesos de selección otorgan a la PSU de matemáticas en la carrera de medicina. Aguirre (2012) reporta que en la carrera de Ingeniería, la prueba más importante para explicar el rendimiento académico en el primer semestre es la PSU de Ciencias, y que en una menor medida, las notas de enseñanza media (NEM) y la PSU de Matemática tienen significancia, pero poca capacidad para discriminar a los mejores estudiantes. Ramírez y Fuentes (2013) ratifican mediante ecuaciones estructurales la relación positiva y significativa entre los indicadores de selección y el rendimiento académico de los estudiantes de Ingeniería Comercial, y que la felicidad tiene un impacto en los indicadores de selección y el rendimiento académico de los mismos.

Recogiendo la información de la situación actual sobre la materia, este estudio se propone analizar los determinantes del éxito de los estudiantes de la Carrera de Ingeniería Comercial - Coquimbo de la Universidad Católica del Norte en Chile. El objetivo es proporcionar herramientas para mejorar el conocimiento de las causales de deserción y del desempeño de los estudiantes a lo largo de su vida estudiantil, y avanzar en la elaboración de estrategias de selección de postulantes con mayor capacidad predictiva del éxito.

\section{METODOLOGÍA}

La variable a explicar es la situación académica de los estudiantes universitarios (Situación) definida como una variable dicotómica, en el cual el éxito es 1 y el fracaso es 0 . Para ello se califica como éxito a los estudiantes titulados o egresados, es decir aquellos que cumplieron con todas las exigencias académicas establecidas por la carrera. Los titulados y los egresados se considera que están en el mismo nivel de éxito debido a que la única diferencia entre ellos es realizar un trámite administrativo y el pago de los derechos para obtener la certificación de la obtención del título. El egresado ha cumplido con todas las exigencias académicas: aprobación de totalidad de los cursos de la malla curricular, aprobación de actividad de titulación y de la práctica pre-profesional. El fracaso engloba a los estudiantes que por algún motivo no concluyeron sus estudios, debido por ejemplo a la eliminación académica, renuncia a la universidad, traslado de universidad o cambio de carrera.

La base de datos en este estudio incluye estudiantes que ingresaron entre los años 1993-1999 a la carrera de Ingeniería Comercial de la Universidad Católica del Norte - Sede Coquimbo, Chile. Para el estudio, además de la variable a explicar (Situación), se han utilizado 13 variables explicativas del éxito o controles, las cuales se pueden clasificar en tres grupos. Éstas se detallan a continuación, y se incluye el nombre de la variable que se usó para el análisis estadístico posterior.

\section{1.- Características Personales}

a) Género (1=masculino y $2=$ =femenino): Género

b) Región (1=Coquimbo, 2=Atacama y Valparaíso, 3=Otra): Región

c) Desfase en años de la educación media (año de ingreso a la carrera restado el año de egreso de la educación media menos 1.0). Por ejemplo, si se ingresa el año siguiente al año de egreso de la educación media, el desfase es cero: Desfase

d) Orden de preferencia en la postulación (1=si fue la carrera de Ing. Comercial - UCN su primera opción de postulación, 2=segunda opción, 3=tercera opción, 4=otra opción): Preferencia

e) Año de Ingreso a la Carrera (1993, 1994, 1995, 1996, 1997, 1998, 1999): Agno 


\section{2.- Formación previa (enseñanza media):}

a) Tipo de colegio en que finalizó la enseñanza media (1=humanista científico, 1=técnico profesional, $3=a m b a s$ modalidades simultáneamente): Tipo_media

b) Tipo de pago ( $1=$ colegios particulares pagados, $2=$ colegios particulares subvencionados, $3=$ colegios municipales): Tipo_pago

c) Puntaje equivalente a notas enseñanza media (335 a 800 puntos, que equivale a las NEM transformadas a un puntaje equivalente): Puntaje_notas

3.- Resultados en la Prueba de Aptitud Académica (PAA). En la actualidad la prueba que selecciona el ingreso de estudiantes universitarios a las universidades tradicionales en Chile es la Prueba de Selección Universitaria (PSU), sin embargo para los estudiantes que conforman nuestra base de datos la prueba de selección era la Prueba de Aptitud Académica (PAA).

a) Puntaje en la PAA Matemáticas : Paa_matemáticas

b) Puntaje en la PAA Verbal : Paa_verbal

c) Puntaje promedio en la PAA : Promedio_paa

d) Puntaje en la prueba de historia y geografía de Chile: Paa_historia

e) Puntaje promedio de selección (una ponderación entre equivalencia de notas de enseñanza media, PAA verbal, PAA matemáticas y prueba de historia y geografía de Chile). Además se consideraba un premio en puntos dependiendo del orden en el cual se postulaba a la carrera: para primera opción 30 puntos, en segunda opción 20 puntos y en tercera opción 10 puntos): Puntaje_selección

La Tabla 1 muestra que la base de datos considera un total de 564 estudiantes. La información incluye los datos de los estudiantes ingresados a la Universidad entre los años 1993 a 1999, ya que para ese grupo a la fecha del estudio se tuvo bastante claridad de su estado hasta el año 2007, es decir si egresaron, se titularon o no. Esta variable aparece identificada en la Tabla con el nombre de Situación.

Tabla 1: Estadísticas resumen de la base de datos

\begin{tabular}{|lccccc|}
\hline Series & Obs & Media & Error Est. & Mínimo & Máximo \\
\hline Situación & 564 & 0,5301 & 0,4995 & 0,0 & 1,0 \\
Agno & 564 & 1996,07 & 1,9707 & 1993,0 & 1999,0 \\
Género & 564 & 1,4823 & 0,5001 & 1,0 & 2,0 \\
Tipo_media & 564 & 1,2908 & 0,5905 & 1,0 & 3,0 \\
Tipo_pago & 564 & 2,1560 & 0,6423 & 1,0 & 3,0 \\
Desfase & 564 & 1,3209 & 2,1862 & 0,0 & 24,0 \\
Región & 564 & 1,3989 & 0,7017 & 1,0 & 3,0 \\
Puntaje_notas & 564 & 574,23 & 90,38 & 335,0 & 785,0 \\
Paa_verbal & 564 & 596,50 & 60,53 & 406,0 & 796,0 \\
Paa_matemáticas & 564 & 627,83 & 60,63 & 466,0 & 801,0 \\
Promedio_paa & 564 & 612,17 & 45,34 & 518,5 & 775,5 \\
Paa_historia & 564 & 571,71 & 75,077 & 381,0 & 755,0 \\
Puntaje_selección & 564 & 621,82 & 39,36 & 545,1 & 773,7 \\
Preferencia & 564 & 1,36 & 0,7 & 1,0 & 4,0 \\
\hline
\end{tabular}

En base a la información presentada en la Tabla 1 se puede indicar algunas características de las variables de análisis. En primer lugar, el porcentaje promedio global de éxito alcanza el $53 \%$. Los datos muestran que existe una leve mayoría masculina (una media de 1,5 en la variable Género corresponde a una proporción 50-50), y dado que la proporción observada es de 1,4823, y entonces hay una cantidad levemente mayor de hombres que de mujeres. La mayoría de los estudiantes provienen de la región de Coquimbo. La prueba de aptitud académica en matemáticas es la que arroja un mejor desempeño por parte de los estudiantes, con un promedio de 627,8 puntos, mientras que la prueba con menor puntaje es la de historia, con 571,7 puntos promedio. También es interesante notar que la gran mayoría de los estudiantes postulan en primera preferencia a esta carrera. 


\section{RESULTADOS}

Como primera aproximación a los determinantes del éxito de los estudiantes se estiman los coeficientes de correlación de Spearman entre el éxito y cada una de las variables explicativas. La Figura 1 muestra las variables ordenadamente en el eje horizontal de acuerdo a su grado de correlación con la variable explicada. Puede observarse que el éxito se relaciona de manera directa y relativamente elevada con las notas de la enseñanza media. Esto es consistente con el estudio de validez predictiva de la PSU elaborado por el Comité Técnico Asesor, H. Consejo de Rectores de las Universidades Chilenas (2006 y 2010), el que indica que las notas de enseñanza media son el factor más eficaz para predecir el rendimiento de los alumnos que ingresan a la universidad. En dicho estudio se reporta que las notas pueden explicar casi un $10 \%$ del desempeño de los estudiantes en el primer año de educación superior, la PSU de matemáticas un $5 \%$ y la PSU de lenguaje casi nada. Este resultado también es reportado en Chile por Pérez et al. (2011). En efecto, la Figura 1 también muestra que el puntaje en la PAA de matemáticas tiene una relación positiva con el éxito, mientras que la PAA verbal tiene una relación negativa.

En conjunto, las notas de enseñanza media junto a ambas pruebas de selección constituyen el puntaje final de aceptación de los estudiantes a la carrera (Puntaje_selección), el que muestra una relación menor a las notas por si solas, al estar afectado hacia la baja por las dos tipos de PAA. Por otra parte, el escaso nivel predictivo de la PAA verbal (incluso inverso) observado en la Figura 1, no parece ser un fenómeno atribuible sólo al hecho que los estudiantes de Ingeniería Comercial posean competencias diferentes a las requeridas con los estudios de la carrera de Ingeniería Comercial, sino que más bien el problema de la PAA verbal es transversal a todo el sistema universitario. Debe notarse también que de acuerdo a Contreras et al. (2009), el haber estado entre los mejores estudiantes en la enseñanza media predice un mejor desempeño universitario en comparación a la PSU y las NEM.

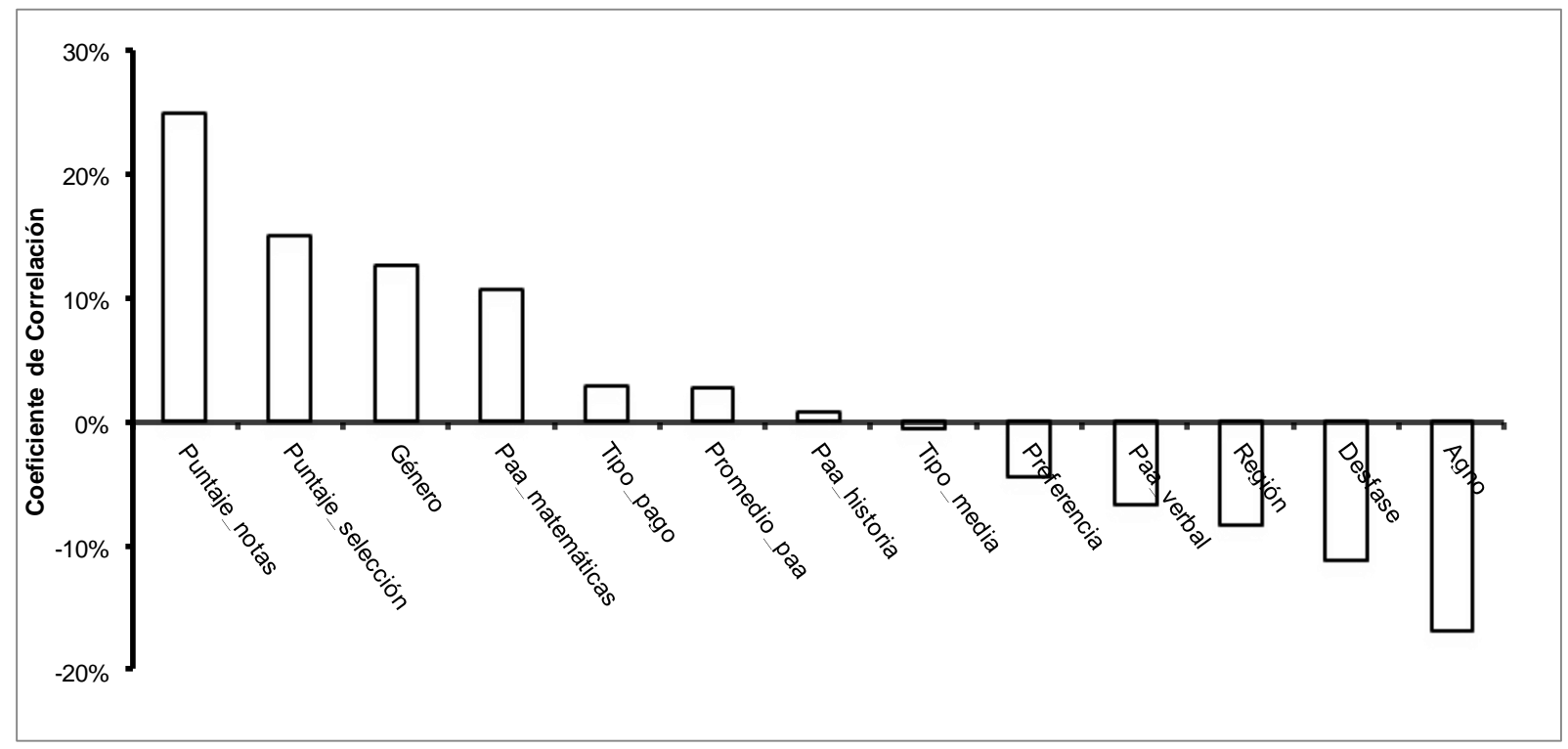

Fig. 1: Correlación entre el éxito y las variables explicativas

Es interesante notar también que el género (Género) muestra una relación directa con el éxito de los estudiantes, siendo las mujeres quienes obtienen una mayor tasa de éxito. Al respecto, Pérez et al. (2011) observan inicialmente una relación similar, pero al realizar pruebas estadísticas específicas en base a un contraste bilateral no logran encontrar diferencias estadísticamente significativas. Por el contrario, Aguirre (2012) encuentra que entre las variables no académicas el sexo es significativo a favor de las mujeres en algunos grupos de cursos solamente. En consecuencia, el género parece ser una variable demasiado ruidosa para ser considerada como predictiva del logro. En el siguiente apartado se analiza este punto con más detalle y se realizan pruebas estadísticas.

El año de ingreso a la carrera muestra una relativamente alta correlación inversa, lo que se explica fundamentalmente en que las tasas de titulación entre las distintas promociones de estudiantes no ha sido la misma. En efecto, quienes ingresaron en el año 1994, por ejemplo, presentaron mayores niveles de logro que los ingresados en el año 1999 (ver Figura 2). Luego, esta observación obedece a un factor de diferencias inter generacionales, y las causas que gatillan dichos resultados probablemente obedecen a una serie de factores motivacionales y de dinámicas de sinergia generadas dentro de la Escuela, cuyos orígenes van más allá de los objetivos de este estudio. La Figura 2 muestra el número de estudiantes ingresados cada año, y el resultado promedio de su éxito medido como porcentaje. 


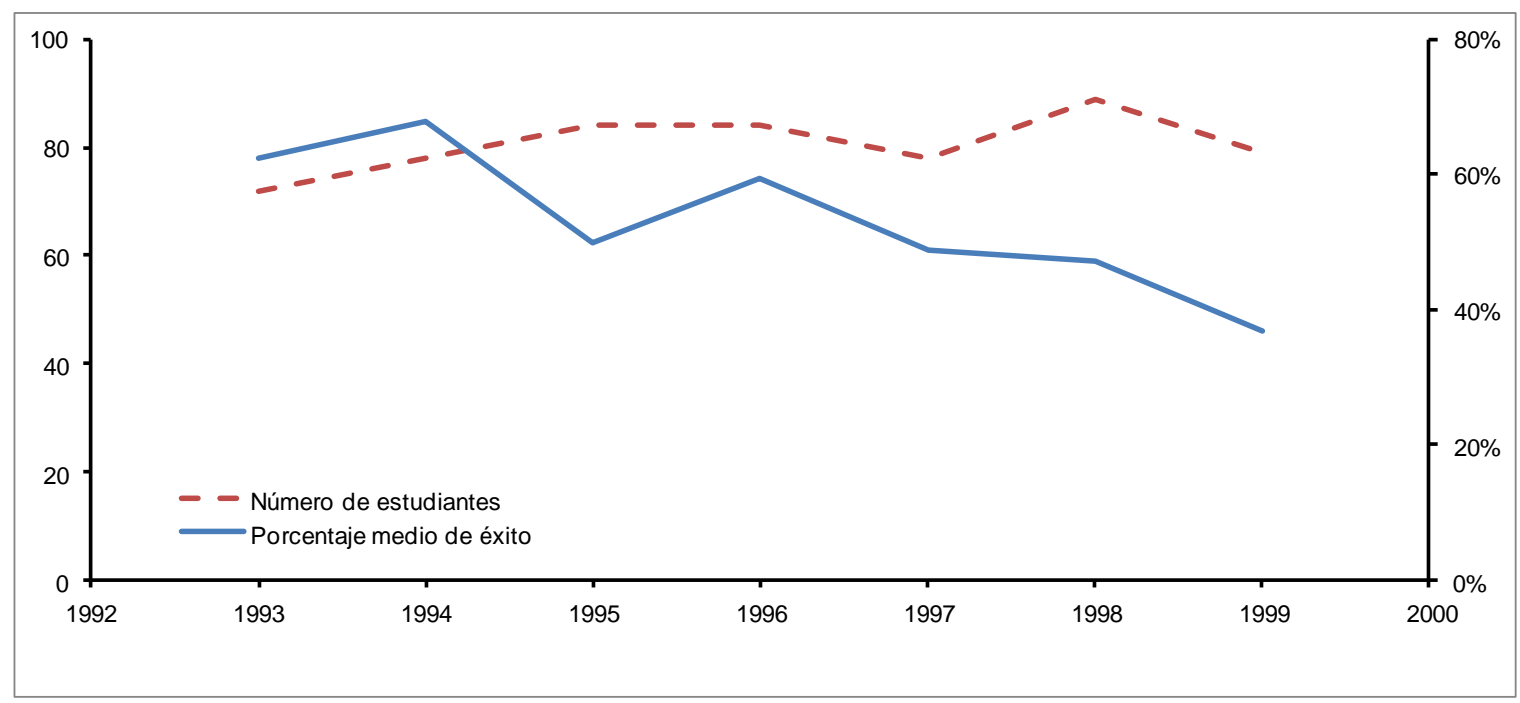

Fig. 2: Grado de éxito de las distintas promociones de estudiantes

La figura 1 muestra también que los años de desfase en los estudios parece ser también una variable determinante, en el sentido de que en la medida que aumentan los años entre el año de egreso de la enseñanza media y el ingreso a la carrera, el éxito es menor. También se puede apreciar que en cuanto el estudiante proviene de regiones más lejanas a la de localización de la Escuela, en promedio el logro es menor. La preferencia de la carrera es otro elemento que actúa de forma inversa, y es que cuando la opción de postulación de estudiante es lejana a las primeras opciones, la tendencia al éxito se ve perjudicada.

Si bien el análisis de correlaciones que se ha hecho anteriormente resulta ilustrativo, un estudio probabilístico es necesario para analizar con mayor detalle las relaciones entre las variables. Puesto que la variable explicada (el éxito) es una variable del tipo dicotómica, entonces resulta apropiado utilizar una especificación del tipo logística (Logit), o modelos de elección cualitativa o binaria. Puesto que en nuestro caso existen sólo dos opciones, el modelo toma la siguiente forma:

$P\left(Y_{i}=1 \mid X_{i}\right)=F\left(X_{i} \beta\right)$

$F(z)=\frac{\exp (z)}{1+\exp (z)}$

donde $Y_{i}$ es la variable dependiente que toma el valor cero o uno, y $X_{i}$ son las variables explicatorias. Los coeficientes de la regresión logit se interpretan como cambios en probabilidades. La tarea es estimar los parámetros $\beta$, pero puesto que son no lineales en $\beta$, la especificación de mínimos cuadrados ordinarios no es apropiada, y debe usarse métodos no lineales, requiriéndose efectuar un ajuste a través de métodos de máxima verosimilitud (maximum likelihood), siendo el método más usado el de Newton-Raphson, puesto que la función objetivo está casi siempre muy bien comportada. Sin embargo lo anterior implica que los supuestos de la distribución son críticos.

Dos son los modelos a analizar en el caso de este estudio:

\section{Modelo 1: Básico.}

En este caso se plantean como variables explicativas los años de desfase en los estudios, el puntaje de notas de la enseñanza media y el resultado de la PAA de Matemáticas, es decir

$$
\operatorname{Prob}\left(\text { Situación=1 } \mid \begin{array}{c}
\text { Desfase } \\
\text { Puntaje_Notas } \\
\text { Paa_Matemáticas }
\end{array}\right)
$$




\section{Modelo 2: Ampliado.}

Aquí adicionalmente a las variables consideradas en el modelo 1, se plantea que la región de la cual provenga el estudiante, el tipo de pago por la educación media y el género de los estudiantes, son variables de las cuales se espera un aporte significativo al éxito.

$\operatorname{Prob}\left(\begin{array}{l|c}\text { Situación=1 } & \begin{array}{c}\text { Desfase } \\ \text { Puntaje_Notas } \\ \text { Paa_Matemáticas } \\ \text { Región } \\ \text { Tipo_Pago } \\ \text { Género }\end{array}\end{array}\right)$

La Tabla 2 muestra los resultados de la estimación del modelo 1. La significancia estadística muestra que los signos de los coeficientes son los esperados, y que todos los coeficientes son confiables a los niveles de error habituales $(5 \%)$, de modo que la estimación del modelo es satisfactoria. De este modo puede decirse entonces que las variables Desfase, Puntaje_notas y PAA_matemáticas explican significativamente la probabilidad de terminar de un modo satisfactorio los estudios de Ingeniería Comercial: a menor desfase se espera un mayor grado de éxito (signo - del coeficiente), y a mayores puntajes de notas en la enseñanza media (signo + del coeficiente) y a mayor puntaje en la PAA de matemáticas (signo + del coeficiente) se aumentan estadísticamente las probabilidades de logro. Adicionalmente, la Tabla 2 muestra que el valor de los coeficientes indica que cada año adicional de desfase de los estudios reduce la probabilidad de éxito un $10 \%$. También, que un puntaje promedio de notas de enseñanza media mayor en 10 puntos genera un incremento en la probabilidad de terminar la carrera de $5.7 \%$. De un modo similar, un puntaje promedio en la PAA de matemáticas mayor en 10 puntos genera un incremento en la probabilidad de terminar la carrera de $4.3 \%$.

Tabla 2: Resultados de la estimación del Modelo 1

\begin{tabular}{|c|c|c|c|c|}
\hline \multicolumn{2}{|c|}{ Logit - Estimation by Newton-Raphson } & & & \\
\hline Convergence in & 4 Iterations & & & \\
\hline Dependent Variable & Situacion & & & \\
\hline Usable Observations & 564.0000 & & & \\
\hline Degrees of Freedom & 560.0000 & & & \\
\hline Cases Correct & 350.0000 & & & \\
\hline Log Likelihood & -365.8175 & & & \\
\hline Average Likelihood & 0.5228 & & & \\
\hline Pseudo-R^2 & 0.0844 & & & \\
\hline Variable & Coef. & Error Est. & T-Stat & Signif \\
\hline Constant & -5.6988 & 1.1360 & -5.0165 & 0.0000 \\
\hline Desfase & -0.1004 & 0.0475 & -2.1154 & 0.0344 \\
\hline Puntaje_notas & 0.0057 & 0.0010 & 5.5240 & 0.0000 \\
\hline Paa_matemáticas & 0.0043 & 0.0015 & 2.8918 & 0.0038 \\
\hline
\end{tabular}

La Tabla 3 muestra los resultados de las estimaciones del Modelo 2. Aquí puede verse que las tres variables incorporadas (Región de procedencia, Tipo de Pago de la Educación y Género) no resultan estadísticamente significativas, por cuanto su significancia excede el valor típicamente usado de 0.05 .

La evidencia reunida acerca de este modelo indica que respecto a la procedencia de los estudiantes, el tipo de financiamiento de los colegios y del sexo, nada puede decirse con razonable confianza acerca de su desempeño futuro en la carrera. Sin embargo, como hemos visto en el modelo 1, queda bastante claro que el puntaje de notas en primer lugar, el resultado de la PAA de matemáticas en segundo lugar, y el desfase en años en tercer lugar, sí logran efectivamente predecir el desempeño universitario. De este modo, los resultados reportados parecen ser consistentes con la teoría, y con la mayor parte de evidencia recogida en estudios previos. 
Tabla 3: Resultados de la estimación del Modelo 2

\begin{tabular}{|c|c|c|c|c|}
\hline \multicolumn{2}{|c|}{ Logit - Estimation by Newton-Raphson } & & & \\
\hline Convergence in & 4 Iterations. & & & \\
\hline Dependent Variable & Situación & & & \\
\hline Usable Observations & 564.0000 & & & \\
\hline Degrees of Freedom & 557.0000 & & & \\
\hline Cases Correct & 362.0000 & & & \\
\hline Log Likelihood & -363.6383 & & & \\
\hline Average Likelihood & 0.5248 & & & \\
\hline Pseudo-R^2 & 0.0919 & & & \\
\hline Variable & Coef. & Error Est. & T-Stat & Signif \\
\hline Constant & -6.3449 & 1.2550 & -5.0557 & 0.0000 \\
\hline Desfase & -0.1048 & 0.0492 & -2.1278 & 0.0334 \\
\hline Puntaje_notas & 0.0050 & 0.0011 & 4.4773 & 0.0000 \\
\hline Paa_matemáticas & 0.0050 & 0.0015 & 3.2585 & 0.0011 \\
\hline Región & -0.1461 & 0.1300 & -1.1235 & 0.2612 \\
\hline Tipo_pago & 0.2106 & 0.1440 & 1.4630 & 0.1435 \\
\hline Género & 0.2387 & 0.1897 & 1.2580 & 0.2084 \\
\hline
\end{tabular}

A nivel latinoamericano otros estudios han demostrado la relevancia de una serie de variables, tales como las calificaciones de la enseñanza secundaria, el género, la educación de los padres e ingreso familiar del estudiante. La literatura muestra que algunas variables resultan ser poco claras, como por ejemplo el género de los estudiantes, el nivel educacional de los padres y el ingreso familiar. Como resultados del estudio, el análisis de correlaciones proporciona algunos resultados iniciales, destacando la alta relación entre los resultados académicos y las notas de enseñanza media, el puntaje de selección, el género, la PAA de matemáticas el año de ingreso, y el desfase entre el término de la enseñanza media y el ingreso a la carrera. Sin embargo, la estimación de un modelo logístico termina por confirmar que sólo las variables notas de enseñanza madia, PAA de matemáticas y años de desfase tienen un poder explicativo del éxito significativo estadísticamente, mientras que las demás variables deben ser dejadas de lado.

Una de las medidas no consideradas aquí, por no encontrarse disponible para los estudiantes analizados, es la medida de habilidad relativa (o ranking) que compara al alumno respecto de sus pares, corrigiendo por el desenvolvimiento en un mismo contexto y entorno. La literatura en Chile sugiere que éste puede ser incluso un mejor predictor del desempeño. Este llamado "ranking de notas" ha sido recientemente introducido en Chile para las casi 80 mil vacantes de las 33 instituciones de educación superior que participan del Proceso de Admisión 2014.

\section{CONCLUSIONES}

El análisis efectuado en este estudio consideró una visión del éxito de los estudiantes en el largo plazo, midiendo el desempeño completo de los estudiantes durante todos los años de la carrera, y definiendo el éxito como el hecho de titularse o estar en la calidad de egresado de la carrera. Esto último implica haber cumplido con todos los requisitos académicos exigidos dentro del plan de estudios de la carrera.

Los resultados obtenidos confirman la tendencia central en la literatura consultada, incluyendo a Bartual y Poblet (2009), en el sentido de que efectivamente el desempeño previo de los estudiantes en la enseñanza media sí tiene poder predictivo respecto del desempeño de los estudiantes. En efecto, las principales variables que resultaron determinantes estadísticamente en el éxito de los estudiantes fueron, en primer lugar las notas de enseñanza media, en segundo lugar el puntaje obtenido en la Prueba de Aptitud Académica de Matemáticas, y finalmente el número de años de desfase entre el año de egreso de la enseñanza media y el año de ingreso a la universidad. 
Las notas de enseñanza media efectivamente parecen indicar que estudiantes con buen desempeño académico antes de entrar a la universidad tienen mejores métodos de estudio, cierta disciplina y responsabilidad individual, características que muestran ser valoradas en el sistema universitario chileno. En este sentido, la importancia de esta variable es consistente con la intuición general. Respecto al puntaje en Prueba de Aptitud Académica de Matemáticas parece tener mayor importancia que la Prueba de Aptitud Académica Verbal, a lo menos en la carrera de Ingeniería Comercial, de modo que la habilidad para resolver problemas relacionados con el razonamiento aritmético, algebraico y geométrico son de relevancia en esta carrera universitaria. Finalmente, los años de desfase entre el egreso de la enseñanza media y el ingreso a la universidad abre una línea de investigación que puede ser estudiada a futuro. Posibles explicaciones a la importancia de esta variable en el desempeño pueden encontrarse en una falta de madurez, desconocimiento de las reales habilidades y competencias al momento de ingresar a la universidad, el no tener las motivaciones suficientes, o la existencia de brechas relevantes entre los perfiles de las carreras y la expectativa que los estudiantes tienen al ingresar a ellas.

Vincular la capacidad de los estudiantes para egresar y/o titularse de una carrera universitaria con los mecanismos de selección inicial es un elemento de gran relevancia, tanto para los estudiantes como para las propias universidades. Globalmente la principal contribución radica en proveer elementos para mejorar la batería de instrumentos de selección de los estudiantes por parte de los centros de estudios superiores de Chile, proporcionando un enfoque metodológico que puede ser aplicado recursivamente en el futuro para una mejor calibración.

\section{REFERENCIAS}

Albalate, D., X. Fageda y J. Perdiguero, Éxito Académico, Características Personales y Proceso de Bolonia: Una Aplicación Econométrica, Revista d'Innovació Docent Universitària: 3, 11-25 (2011).

Aguirre, N., Factores Que Predicen El Rendimiento Académico En La Escuela De Ingeniería De La Universidad De Chile. Tesis de Magíster, Dpto. de Ingeniería Industrial, Universidad de Chile, Santiago, Chile (2012).

Bastías, G., L. Villarroel, D. Zúñiga, G. Marshall, N. Velasco y B. Mena, Desempeño Académico de Los Estudiantes De Medicina: ¿Un Resultado Predecible?, Revista Médica de Chile: 128 (6), 671-678, (2000).

Bartual, Teresa y M. C. Poblet, Determinantes del rendimiento académico en estudiantes universitarios de primer año de Economía, Revista de Formación e Innovación Educativa Universitaria: 2(3), 172-181 (2009).

Chain, R., N. Cruz, N., M. Martínez, M. y N. Jácome, Examen de Selección de Probabilidad de Éxito Escolar en Estudios Superiores. Estudio en una universidad pública estatal mexicana, Revista Electrónica de Investigación Educativa: 5(1), 1-17, (2003).

Comité Técnico Asesor, H. Consejo de Rectores de las Universidades Chilenas, Validez Predictiva de los Factores de Selección a las Universidades del Consejo de Rectores, (2006), http://www.consejoderectores.cl/web/pdf/validez_diferencial.pdf. Acceso: 15 de Marzo (2014).

Comité Técnico Asesor, H. Consejo de Rectores de las Universidades Chilenas, Resultados de la Aplicación de Pruebas de Selección Universitaria Admisión 2006_2010 (2010), http://www.consejoderectores.cl/web/pdf/resultados_admisiones2006_2010.pdf, Acceso: 15 de Marzo (2014).

Contreras, D., S. Gallegos, y F. Meneses, Determinantes de Desempeño Universitario: ¿Importa la habilidad relativa? Análisis ex-ante y ex-post de una política pública, Informe final. Santiago: CNE (2009).

Duarte, M. y J. Galaz, J, Predicciones del desempeño académico en el $1^{\circ}$ año de universidad en una institución pública estatal (Universidad Autónoma de Baja California), 6ㅇ Congreso Internacional Retos y Expectativas de la Universidad: El Papel de la Universidad en la Transformación de la Sociedad, Puebla, México, (2006).

Herrera García, M.E., S. Nieto Martín. M.J. Rodríguez Conde y M. Cruz Sánchez Gómez, Factores Implicados en el Rendimiento Académico de los Alumnos Universidad de Salamanca, Revista de Investigación Educativa: 17(2), 413-421, (1999).

Huesca, M. y M. Castaño, Causas de la Deserción en Estudiantes Universitarios de 1ํAño de una Universidad Privada, Revista Mexicana de Orientación Educativa: V (12), 34-39, (2007). 
Ishitani, T. y K.G. Snider, Longitudinal Effects Of College Preparation Programs On College Retention, Association for Institutional Research: 9(3), 1-10 (2006).

Ibarra, M. y J. Michalus, Análisis del Rendimiento Académico Mediante un Modelo Logit, Ingeniería Industrial: 9(2), 47-56, (2010).

Martínez-Padilla, J. y J. A. Pérez-González, Efecto de la Trayectoria Académica en el Desempeño de Estudiantes de Ingeniería en Evaluaciones Nacionales, Formación Universitaria: 1(1), 3-12 (2008).

Pérez, C., L. Ortiz y P. Parra, Prueba De Selección Universitaria, Rendimiento en Enseñanza Media y Variables Cognitivo-Actitudinales de Alumnos De Medicina, Revista Educación en Ciencias de la Salud: 8(2), 120-127, (2011).

Ramírez, P. y C. Fuentes, Felicidad y Rendimiento Académico: Efecto Moderador de la Felicidad sobre Indicadores de Selección y Rendimiento Académico de Alumnos de Ingeniería Comercial, Formación Universitaria: 6(3), 21-30, (2013).

Reyes, A. y M. Torres Pavez. La PSU y otros Factores de Rendimiento y Éxito Académico Universitario. El caso de la Pontificia Universidad Católica de Valparaíso, Memoria de Titulación, Escuela de Ingeniería Comercial, Pontificia Universidad Católica de Valparaíso, Valparaíso, Chile, (2009).

Romo, A., y M. Fresán, Los Factores Curriculares y Académicos Relacionados con el Abandono y el Rezago, (2001), http://proyectodeintervencion-ca2.wikispaces.com/file/view/docto+3.pdf, Acceso: 10 Enero (2014).

Tinto, V., Leaving College: Rethinking the Causes and the Cures of Student Attrition, University of Chicago Press, 312, Chicago, EEUU, (1993).

Vásquez, F. y J. Cabrera, Algunos Determinantes del Éxito en Completar el Bachillerato: Estudio Longitudinal de 6 Años, Universidad de Puerto Rico en Cayey, Puerto Rico, (2004). 